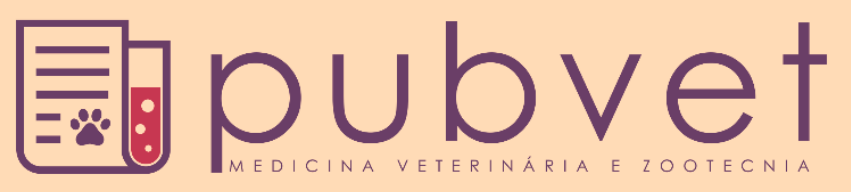

https://doi.org/10.31533/pubvet.v13n3a299.1-6

\title{
Colecistite supurativa crônica associada a obstrução de ducto biliar comum em felino: Relato de caso
}

\author{
Gisandra de Fátima Stangherlin ${ }^{1} \bullet$, Veridiane da Rosa Gomes ${ }^{1}{ }^{\bullet}$, Aparício Mendes de \\ Quadros $^{10}$, Carla Reis ${ }^{2}{ }^{\circ}$, Heloísa Helena de Alcântara Barcellos ${ }^{3}{ }^{\circ}$, Marco Augusto

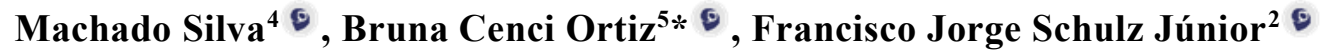 \\ ${ }^{1}$ Médica Veterinária Residente de Clínica Médica e Cirurgia de Pequenos Animais do Hospital Veterinário da Universidade de Passo Fundo - RS/Brasil. \\ ${ }^{2}$ Acadêmico(a) do curso de Medicina Veterinária da Universidade de Passo Fundo - RS/Brasil. \\ ${ }^{3}$ Professora Mestre do curso de Medicina Veterinária da Universidade de Passo Fundo, Departamento de Clínica de Pequenos Animais - RS/Brasil. \\ ${ }^{4}$ Professor Doutor do curso de Medicina Veterinária da Universidade de Passo Fundo, Departamento de Cirurgia de Pequenos Animais - RS/Brasil \\ ${ }^{5}$ Médica Veterinária Residente de Clínica Médica de Pequenos Animais do Hospital Veterinário da Universidade Lutera do Brasil - Canoas/RS-Brasil. \\ *Autor para correspondência, E-mail: bruna.ortizvet@hotmail.com
}

\begin{abstract}
Resumo. O objetivo deste trabalho foi relatar um caso de obstrução de ducto biliar comum decorrente de colecistite crônica em um felino fêmea, sem raça definida, com quatro anos de idade. A colecistite caracteriza-se por uma inflamação do trato biliar que, em alguns gatos, pode também estender-se ao parênquima hepático, sendo uma das principais causas de obstrução do ducto biliar. Outras causas comuns de obstrução das vias biliares estão relacionadas com pancreatites e colangites. Os sinais clínicos são inespecíficos e sua manifestação depende do tempo e da intensidade da obstrução (total ou parcial), normalmente apresentam icterícia, anorexia, vômito, diarreia e perda de peso. O diagnóstico definitivo costuma ser realizado por meio do exame ultrassonográfico, que revela dilatação do trato biliar e da vesícula biliar. Também é esperado aumento na atividade das enzimas hepatobiliares, bilirrubina, fosfatase alcalina, gama-glutamil transferase e colesterol em função da colestase. Como tratamento preconiza-se nos casos de obstrução parcial o tratamento clínico e nos casos de obstrução total ou na ausência de melhora com o tratamento clínico a coledocoduodenostomia e o prognóstico depende da causa e grau da obstrução. Quando necessária a intervenção cirúrgica o prognóstico se torna desfavorável, principalmente pela debilidade do paciente e risco de desenvolvimento de peritonite no pós-operatório.
\end{abstract}

Palavras chave: coledocoduodenostomia, gatos, hiperproteinemia, icterícia, parênquima hepático

\section{Cholecystitis chronic suppurative associated with obstruction of biliary duct common in feline: Case report}

Abstract. The objective of this study was to report a case of common bile duct obstruction due to chronic cholecystitis in a female feline, without defined race with four years old. The cholecystitis characterized by inflammation of the biliary tract, which in some cats may also extend up to the hepatic parenchyma, one of the main causes of obstruction of the biliary bucto. Other common causes of biliary tract obstruction are related to pancreatitis and cholangitis. Clinical signs are nonspecific and its manifestation depends on the time and intensity of the obstruction (total or partial), usually have jaundice, anorexia, vomiting, diarrhea and weight loss. The definitive diagnosis is usually performed by ultrasonography, which reveals dilatation of the biliary tract and gallbladder. Also expect increased activity of hepatobiliary enzymes, bilirubin and cholesterol in the light of cholestasis. As a treatment it is recommended in cases of partial obstruction clinical treatment and in cases of total obstruction or absence of improvement with clinical treatment 
choledochoduodenostomy and the prognosis depends on the cause and degree of obstruction. When required surgery the prognosis becomes unfavorable, mainly by the weakness of the patient and risk of peritonitis development postoperatively.

Keywords: choledochoduodenostomy, cats, hyperproteinemia, jaundice, hepatic parenchyma

\title{
Colecistitis supurativa crónica asociada a obstrucción de conducto biliar común en felino: Reporte de un caso
}

\begin{abstract}
Resumen. El objetivo de este trabajo fue relatar un caso de obstrucción de conducto biliar común derivado de colecistitis crónica en un felino hembra, sin raza definida, con edad de cuatro años. La colecistitis se caracteriza por una inflamación del tracto biliar que, en algunos gatos, también puede extenderse al parénquima hepático, siendo una de las principales causas de obstrucción del conducto biliar. Otras causas comunes de obstrucción de las vías biliares están relacionadas con pancreatitis y colangitis. Los signos clínicos son inespecíficos y su manifestación depende del tiempo y de la intensidad de la obstrucción (total o parcial), normalmente presentan ictericia, anorexia, vómito, diarrea y pérdida de peso. El diagnóstico definitivo suele ser realizado por medio del examen ultrasonográfico, que revela dilatación del tracto biliar y de la vesícula biliar. También se espera un aumento en la actividad de las enzimas hepatobiliares, bilirrubina, fosfatasa alcalina, gammaglutamil transferasa y colesterol en función de la colestasis. Como tratamiento se preconiza en los casos de obstrucción parcial el tratamiento clínico y en los casos de obstrucción total o en la ausencia de mejora con el tratamiento clínico a la coledocoduodenostomía y el pronóstico depende de la causa y grado de la obstrucción. Cuando es necesaria la intervención quirúrgica el pronóstico se vuelve desfavorable, principalmente por la debilidad del paciente y riesgo de desarrollo de peritonitis en el postoperatorio.
\end{abstract}

Palabras clave: coledocoduodenostomía, gatos, hiperproteinemia, ictericia, parénquima hepático

\section{Introdução}

Entre as desordens hepáticas que acometem os felinos, as doenças do trato biliar são as de maior ocorrência (Center, 2009). As obstruções do ducto biliar comum em felinos, ocorrem, geralmente, secundárias a uma doença inflamatória, como pancreatite, colangite e colecistite (Mayhew \& Weisse, 2008). As manifestações clínicas da obstrução do ducto biliar comum são inespecíficas e variam conforme o grau de obstrução (Lehner \& McAnulty, 2010).

A ultrassonografia é a ferramenta de diagnóstico mais útil para diferenciar a obstrução do ducto biliar de outras doenças do trato biliar (Watson \& Bunch, 2009). Além da bilirrubina, a avaliação laboratorial inclui a dosagem das enzimas ALT (alanina aminotransferase), AST (aspartato aminotransferase), FA (fosfatase alcalina) e GGT (gamaglutamiltransferase) (Nelson \& Couto, 2015). Elevações da FA são indicativas de doença hepatobiliar, assim como elevação da GGT nos casos de obstrução biliar, mas também podem se elevar nas doenças hepatobiliares primárias com colestase intra-hepática (Richter, 2005). O tratamento da obstrução do ducto biliar comum depende da causa e se a obstrução é parcial ou total (Nelson \& Couto, 2015). Quando a obstrução é parcial, onde não há presença de fezes acólicas, o tratamento clínico deve ser instituído com coléricos e antioxidantes. Também é fundamental o tratamento da doença de base, se não houver resposta clínica ou aparecerem sinais de obstrução completa, a cirurgia é indicada (Watson \& Bunch, 2009).

Nas obstruções totais do sistema biliar ocorrem alterações fisiológicas progressivas e graves levando o animal à óbito. Para o reestabelecimento do fluxo biliar, é preconizado à intervenção cirúrgica para remoção da obstrução ou realização de um desvio no sistema biliar (coledocoenterostomia ou colecistoenterostomia) como realizado neste paciente. Os procedimentos cirúrgicos requerem habilidade técnica, principalmente pela manipulação de tecidos inflamados em pacientes que podem ter alterações de coagulação (Lehner \& McAnulty, 2010). 


\section{Relato de caso}

Foi atendido no Hospital Veterinário da Universidade de Passo Fundo, um felino fêmea com quatro anos de idade, sem raça definida, com 3,8 kg, apresentando icterícia, hiporexia e vômito há dois meses. A paciente havia sido atendida em outra clínica com suspeita de hemoparasitose, recebeu metoclopramida como tratamento, o qual não foi eficaz para a melhora dos sinais clínicos. Ao exame físico, apresentou discreta hipertermia $\left(39,6^{\circ} \mathrm{C}\right)$, mucosas ictéricas e algia abdominal na região epigástrica. Como exames complementares foram solicitados hemograma, bioquímica sérica (FA, ALT, albumina, ureia e creatinina), esfregaço sanguíneo e ultrassonografia abdominal. Observou-se hiperproteinemia, enzimas hepáticas elevadas (ALT: $626 \mathrm{UL}^{-1}$ e FA: $225 \mathrm{U} \mathrm{L}^{-1}$ ), na ultrassonografia foi observada a presença de estruturas arredondadas e tubulares, tortuosas, anecogênicas e hipoecogênicas, os ductos biliares e vasos hepáticos preservados, imagens sugestivas de obstrução biliar extra hepática ou neoplasia (Figura 1-a).

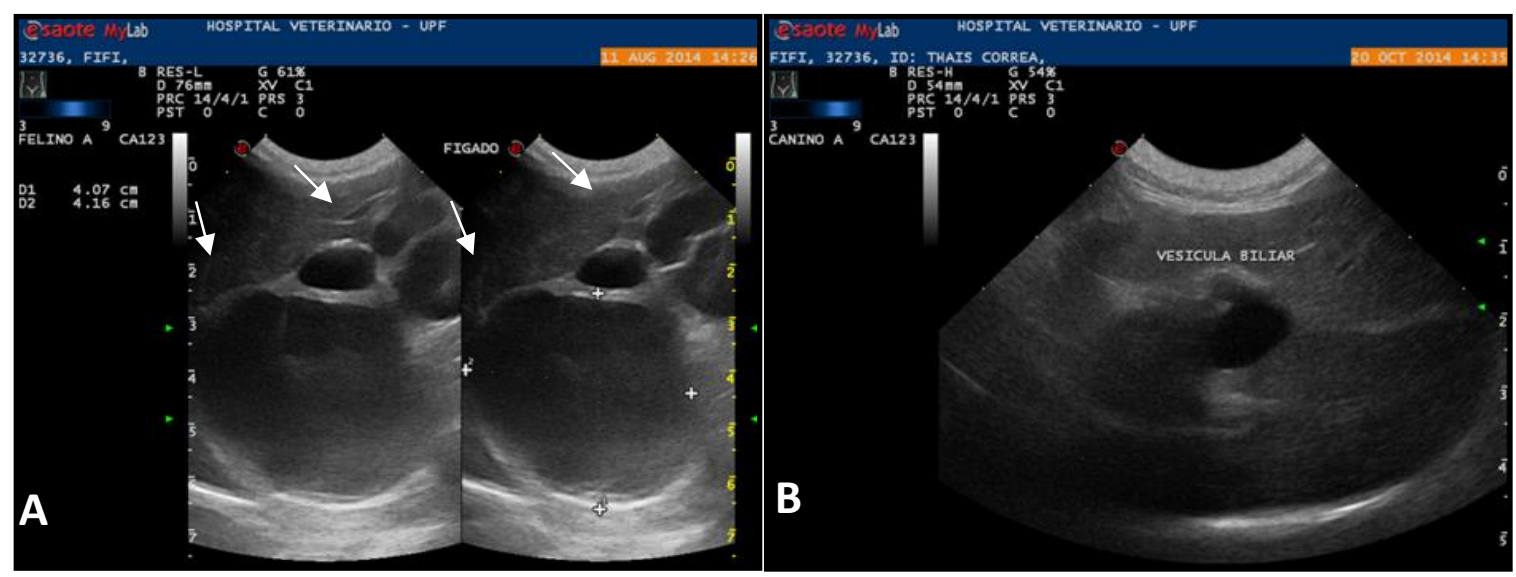

Figura 1. A) Imagem ultrassonográfica mostrando coleções císticas (seta) no parênquima hepático, próximas a vesícula biliar, compatíveis com obstrução ou neoplasia. B) Imagem ultrassonográfíca do fígado e da vesícula biliar de um felino fêmea de 4 anos de idade, 3 meses após a realização de coledocoduodenostomia.

A paciente permaneceu internada por oito dias recebendo cefalotina, metronidazol, ondasentrona, omeprazol, silimarina e ácido ursodesoxicólico. Como não houve melhora clínica e a causa da obstrução não havia sido definida com os exames complementares, a felina foi encaminhada para videolaparoscopia exploratória. Antes do procedimento cirúrgico foram realizados novos exames préoperatórios, onde observou-se que as enzimas hepáticas continuavam elevadas, além de aumento significativo da bilirrubina e GGT. Durante a videocirurgia constatou-se aumento de volume irregular na região da vesícula biliar. O conteúdo biliar foi aspirado por colecistocentese, com agulha fina para análise citológica, no momento de remoção da agulha houve perfuração e extravasamento de líquido na cavidade, necessitando conversão para técnica aberta.

Constatou-se obstrução do colédoco, cerca de $3 \mathrm{~cm}$ da junção coledocoduodenal, ductos biliares hepáticos e císticos gravemente distendidos (Figura 2), então foi realizada a coledocoduodenostomia. Incisionou-se aproximadamente $2 \mathrm{~cm}$ no ducto dilatado na sua porção patente, uma incisão semelhante foi feita no duodeno para a realização da anastomose entre o colédoco e o duodeno. A sutura foi realizada com fio inabsorvível monofilamentar náilon 5-0, padrão contínuo simples. O fragmento do ducto biliar obstruído foi removido e encaminhado para exame histopatológico que resultou no diagnóstico de colangite neutrofílica.

No pós operatório imediato foi administrado tramadol $\left(4 \mathrm{mg} / \mathrm{kg}^{-}\right.$, SC, TID (três vezes ao dia)), cefalotina $\left(30 \mathrm{mg} / \mathrm{kg}^{-}, \mathrm{IV}\right.$, TID), enrofloxacina 2,5\% (2,5 mg/ $\mathrm{kg}^{-}$, IV, BID (duas vezes ao dia)), metronidazol (7,5 mg/kg-, IV, BID), ondasentrona ( $0,22 \mathrm{mg} / \mathrm{kg}^{-}$, IV, BID), omeprazol (1 mg/kg-, IV, SID (uma vez ao dia)), silimarina (30 mg/kg-, VO, SID), ácido ursodesoxicólico (15 mg/kg-, VO, SID) e meloxican $\left(0,1 \mathrm{mg} / \mathrm{kg}^{-}, \mathrm{SC}, \mathrm{SID}\right)$. No dia seguinte a paciente já demonstrou interesse pela ração. A dieta foi baseada em ração comercial com proteína de elevada qualidade, composta de apresentação úmida e seca para estimular a ingestão de alimento, previnindo assim a posibilidade do desenvolvimento da lipidose hepática, principalmente no início do tratamento devido a hiporexia. Após oito dias apresentava 
normorexia, normodipsia e melhora no quadro geral, além da ausência de icterícia, então recebeu alta, onde foi prescrito cefalexina por mais quatro dias, enrofloxacina por mais quatro dias, silimarina por mais quinze dias, omeprazol e ácido ursodesoxicólico por mais sete dias. A paciente retornou quinze dias após a alta para reavaliação, estava ativa e se alimentando normalmente. Neste momento foi realizada também ultrassonografia abdominal que revelou parênquima hepático preservado, contorno regular, presença de formações arredondadas com contorno irregular, com conteúdo anecogênico, próxima à vesícula biliar, compatível com cistos. Já a vesícula biliar estava com formato anatômico preservado. Os exames laboratoriais foram repetidos (bilirrubinas, ALT, FA, GGT e albumina), e se encontravam dentro dos valores de referência para a espécie. A mesma foi acompanhada através de consultas periódicas durante três meses, onde apresentou hábitos alimentares normais e ganho de peso progressivo, também foi realizado acompanhamento ultrassonográfico de região hepática e de vesicula biliar que com o passar dos meses demonstraram imagens anatômicas normais (Figura 1-b).

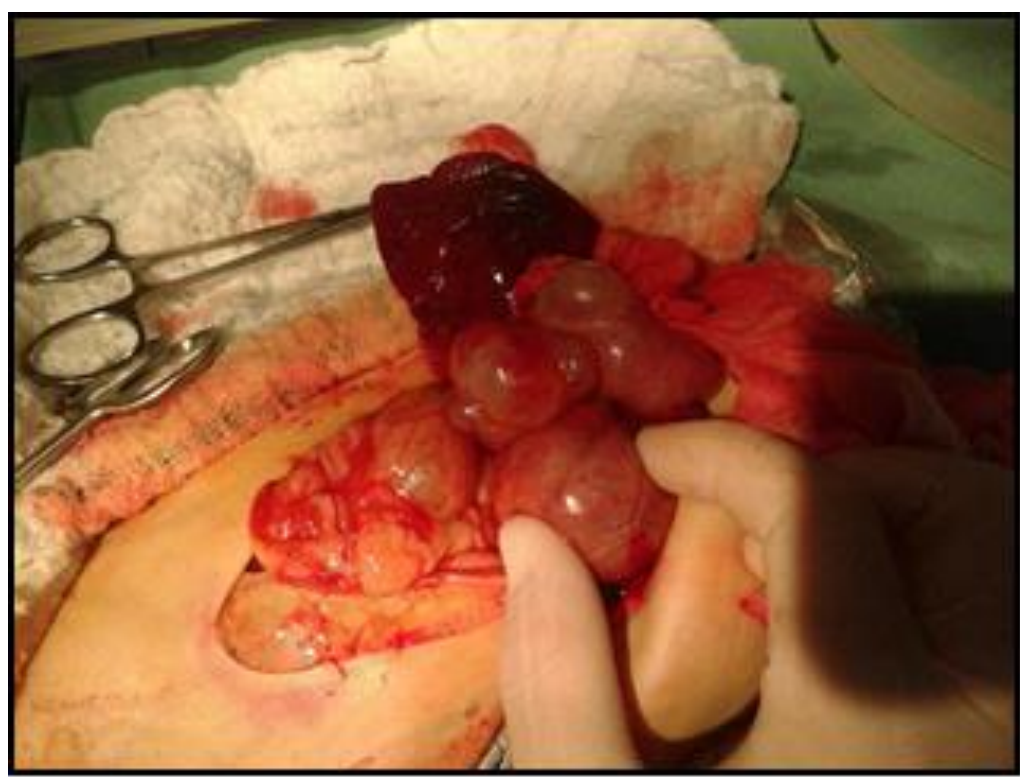

Figura 2. Imagem demonstrando formações císticas em região hepática, decorrentes da obstrução do ducto biliar em uma felina, SRD de 4 anos de idade.

\section{Discussão}

A colecistite neutrofílica é frequentemente observada em gatos, podendo ocorrer isoladamente ou em associação com a colangite neutrofílica (Nelson \& Couto, 2015). No presente caso, o exame histopatólogico revelou colecistite neutrofílica. Entretanto, não foi possível estabelecer se a alteração estava ocorrendo de forma isolada ou associada à colangite. Para melhor esclarecimento sugere-se realizar biopsia hepática. A colecistite também pode ocorrer juntamente ou secundariamente a pancreatite. Neste caso, não foram encontradas alterações bioquímicas e macroscópicas no pâncreas.

A variação dos sinais clínicos está correlacionada ao tipo de obstrução (total ou parcial), complacência do sistema biliar e se os agregados da lama biliar estão obstruindo o ducto biliar comum (Lehner \& McAnulty, 2010). Portanto, se a obstrução for parcial, as manifestações clínicas podem oscilar por várias semanas, o que explica os episódios esporádicos de vômito e letargia a mais de dois meses. Entretanto, mesmo se tratando de uma obstrução parcial, neste caso, optou-se pela realização do procedimento cirúrgico, pois o colédoco estava extremamente tortuoso e a vesícula biliar distendida formando várias coleções císticas armazenando conteúdo biliar, deixando evidente a necessidade da intervenção cirúrgica para a sobrevivência do animal, além disso o tratamento clínico não havia se mostrado eficaz.

A natureza genérica dos sinais clínicos associados à obstrução biliar do ducto comum pode tornar 0 diagnóstico difícil, se for baseada apenas nos sinais clínicos. Por isso, exames laboratoriais e de imagem devem ser solicitados (Lehner \& McAnulty, 2010). Os sinais clínicos e os exames laboratoriais eram compatíveis com obstrução do ducto biliar. Na maioria das vezes, como observado por Mehler \& 
Bennett (2006), o exame ultrassonográfico é usado para o diagnóstico definitivo de obstrução biliar; porém, neste caso as imagens foram sugestivas de obstrução, mas hipóteses como, neoplasias não puderam ser descartadas. Com isso as imagens foram inconclusivas para estabelecer um diagnóstico definitivo e optou-se pela exploração para obtenção do diagnóstico, realizando o tratamento cirúrgico.

A severa icterícia apresentada pela paciente se justifica pelo fato de que a bilirrubina direta se encontrava 3,5 vezes acima do valor de referência, conforme cita Zoran (2011) valores de bilirrubina excedente de $2,5 \mathrm{mg} / \mathrm{dl}$ já seriam suficientes para causar tal sintoma e quando este valor for duas ou três vezes maior, o fígado perde a capacidade de processar o excesso. No retorno, após quinze dias da cirurgia, foi repetida a mensuração das bilirrubinas e das enzimas hepatobiliares, que apresentavam valores dentro da normalidade deixando claro que o procedimento cirúrgico foi eficaz para corrigir a obstrução.

Segundo Center (2009), há aumento das atividades das transaminases parenquimatosas (ALT e AST) e enzimas colestásicas (FA e GGT) na maioria das desordens do sistema biliar, com ou sem hiperbilirrubinemia ou icterícia. Concordando com a literatura houve aumento significativo da ALT e da GGT. O aumento da ALT e AST ocorre em situações de citólise do hepatócito, ou seja, elas extravasam do interior do hepatócito. Já o aumento da FA e GGT decorre da inabilidade que as enzimas têm em serem excretadas pelo sistema biliar, ou seja, em casos de colestase (Richter, 2005).

As cirurgias do trato biliar em felinos estão associadas a diversas complicações fatais, levando a alta mortalidade, sendo realizadas, portanto, somente quando a obstrução for total, visto que o prognóstico das obstruções parciais é bom quando utilizado tratamento clínico (Nelson \& Couto, 2015). Com a visualização da obstrução houve a necessidade do tratamento cirúrgico, pois era preciso reestabelecer o fluxo biliar. Avaliando a obstrução, que se tratava de um ponto de fibrose severa, a técnica empregada foi a coledocoduodenostomia, ou seja, anastomose do colédoco com o duodeno. A porção do ducto que estava obstruída em função da fibrose foi retirada, assim, realizou-se a anastomose da porção viável diretamente no duodeno. As complicações das anastomoses enterobiliares incluem extravasamento do conteúdo biliar, deiscência de pontos e colangite associadas ao refluxo do conteúdo intestinal por drenagem inadequada da vesícula biliar para o intestino (Mehler \& Bennett, 2006).

É esperado um crescimento bacteriano dentro do trato biliar durante o pós-operatório inicial, embora, a colangite clínica geralmente não se desenvolve exceto se o trato biliar tornar-se obstruído ou se a incisão de drenagem for pequena demais (Mehler \& Bennett, 2006). A escolha dos antibióticos deve ser baseada na cultura e/ou literatura que afirma que as principais bactérias envolvidas são de origem gastrointestinal (Gram-positivas, negativas e anaeróbicas) (Nelson \& Couto, 2015). A paciente em questão foi avaliada periodicamente durante a internação, através de exames hematológicos para controle de uma possível peritonite, e não apresentou alterações compatíveis com infecção.

O prognóstico das obstruções biliares que demandam intervenção cirúrgica, segundo a literatura é reservado (Nelson \& Couto, 2015), mas neste caso, foi bem sucedido havendo a melhora clínica da paciente.

\section{Conclusão}

A colecistite supurativa crônica pode ser facilmente confundida com outros processos que causam obstrução de ducto biliar. Dessa forma, faz-se imprescindível a avaliação clínica rigorosa, assim como exames laboratoriais e de imagem, citológicos, biópsias e exames histopatológicos para determinar o diagnóstico e o estabelecimento de uma conduta terapêutica e técnica cirúrgica adequada e eficiente.

\section{Referências bibliográficas}

Center, S. A. (2009). Diseases of the gallbladder and biliary tree. Veterinary clinics of north america: Small animal practice, 39(3):543-598.

Lehner, C. \& McAnulty, J. (2010). Management of extrahepatic biliary obstruction: a role for temporary percutaneous biliary drainage. Compedium Continung Education for Veterinarians, 32(18):E1-E10.

Mayhew, P. D. \& Weisse, C. W. (2008). Treatment of pancreatitis-associated extrahepatic biliary tract obstruction by choledochal stenting in seven cats. Journal of Small Animal Practice, 49(3):133-138. 
Mehler, S. J. \& Bennett, R. A. (2006). Canine extrahepatic biliary tract disease and surgery. Compendium Continung Education for Veterinarians, 28(4):302-314.

Nelson, R. W. \& Couto, C. G. (2015). Medicina interna de pequenos animais. Amsterdan: Elsevier Editora.

Richter, K. P. (2005). Doenças do fígado e do sistema hepatobiliar. In T. R. Tams (Ed.), Gastroenterologia de pequenos animais (pp. 283-348). São Pauylo, Brasil: Roca.

Watson, F. J. \& Bunch, S. E. (2009). Hepatobiliary diseases in cat. In W. R. Nelson \& C. G. Couto (Eds.), Small animal internal medicine. Elsevier mosby, St.Louis, Missouri, U. St.Louis, Missouri, USA: Elsevier Mosby.

Zoran, D. L. (2011). Disease os the liver. In S. E. Little (Ed.), The cat - Clinical Medicine and Management. St.Louis, Missouri, USA: Saunders.

Recebido: 22 de fevereiro, 2019

Aprovado: 15 de março, 2019.

Publicado: 28 de março, 2019.

Licenciamento: Este artigo é publicado na modalidade Acesso Aberto sob a licença Creative Commons Atribuição 4.0 (CC-BY 4.0), a qual permite uso irrestrito, distribuição, reprodução em qualquer meio, desde que o autor e a fonte sejam devidamente creditados. 